where $A$ is a unit-vector (say $A=\cos \lambda+\iota \sin \lambda$ ) and $B, B^{\prime}$ are conjugate vectors. Or, writing $\mathrm{B}=b+\iota \beta, \mathbf{B}^{\prime}=b-\iota \beta$, the constants are $\lambda, b, \beta ; 3$ constants as it should be."

\title{
Quaternion Synopsis of Hertz' View of the Electrodynamical Equations.
}

By Professor TaIT.

\section{Note on Menelaus's Theorem.}

By R. E. Allardice, M.A.

$\$ 1$. The object of this nute is, in the first place, to show that Menelaus's Theorem, regarding the segments into which the sides of a triangle are divided by any transversal, is a particular form of the condition, in trilinear co-ordinates, for the collinearity of three points; and, in the second place, to point out an analogue of Menelaus's Theorem in space of three dimensions.

$\S 2$. In the usual system of areal co-ordinates, the $x$-co-ordinate of $\mathrm{P}$ (fig. 52) is $\triangle \mathrm{PBC} / \triangle \mathrm{ABC}$, that is $\mathrm{PD} / \mathrm{AD}$. Now let $\mathrm{D}, \mathrm{E}, \mathrm{F}$, be three points in $\mathrm{BC}, \mathrm{CA}, \mathrm{AB}$, respectively, dividing these sides in the ratios $l_{1} / m_{1}, l_{2} / m_{2}, l_{3} / m_{3}$; then the co-ordinates of $\mathrm{D}, \mathrm{E}, \mathrm{F}$, are proportional to $\left(0, m_{1}, l_{1}\right),\left(l_{2}, 0, m_{2}\right),\left(m_{3}, l_{3}, 0\right)$. Hence the condition that $\mathrm{D}, \mathrm{E}, \mathrm{F}$, lie on the straight line $\mathrm{A} x+\mathrm{B} y+\mathrm{C} z=0$ is

$$
\left|\begin{array}{ccc}
0 & m_{1} & l_{1} \\
l_{2} & 0 & m_{2} \\
m_{3} & l_{3} & 0
\end{array}\right|=0
$$

that is, $l_{1} l_{2} l_{3}+m_{1} m_{2} m_{3}=0$, which is Menelaus's Theorem.

\$ 3. In space of three dimensions we may use the corresponding system of tetrahedral co-ordinates, and obtain a theorem analogous to that of Menelaus.

Let $\mathrm{BCD}$ (fig. 53 ) be one of the faces of the tetrahedron; and put $a_{2}=\mathrm{PB}^{\prime} / \mathrm{BB}^{\prime}=\Delta \mathrm{PCD} / \mathrm{BCD}, a_{3}=\mathrm{PC}^{\prime} / \mathrm{CC}^{\prime}=\Delta \mathrm{PDB} / \Delta \mathrm{CDB}$, etc. Then the co-ordinates of $P, Q, R, S$, points in the four faces of the tetrahedron, may be written $\left(0, a_{3}, a_{3}, a_{4}\right),\left(b_{1}, 0, b_{3}, b_{4}\right)$, etc. ; and the condition that these four points be coplanar is 